\title{
In Situ Scanning Transmission Electron Microscopy (STEM) of Individual Electrochemical Intercalation Events in Graphite
}

Jared J. Lodico ${ }^{1}$, E. R. White ${ }^{1}$, William A. Hubbard ${ }^{1}$, Erick Garcia ${ }^{1}$, Bradley Parks ${ }^{1}$, Brian Zutter $^{1}$, and B. C. Regan ${ }^{1}$

1. Department of Physics \& Astronomy and California NanoSystems Institute, University of California, Los Angeles, California 90095 USA

Graphite intercalation compounds (GICs) are formed when ions or molecules (intercalants) are inserted between the carbon layers of a graphite host. With some electrolytes a reversible charge transfer process occurs during intercalation, making GICs attractive materials for batteries. The demand for improved batteries has highlighted the need for in situ measurements probing electrode-electrolyte interactions [1]. With in situ scanning transmission electron microscopy (STEM) we observe the reversible electrochemical intercalation of multi-layered $(\sim 20-100$ layers) graphene in $96 \%$ sulfuric acid $\left(\mathrm{H}_{2} \mathrm{SO}_{4}\right)$.

Pristine, natural graphite is most commonly A-B (Bernal) stacked, where every other graphene layer B is horizontally offset from its neighbors A by the carbon-carbon bond length. Upon intercalation the crystal structure changes, and the graphene layers above and below the intercalant layer can shift. These shifts change the intensity of a coherently scattered electron beam. For example, if the GIC adopts the stacking $\mathrm{A}|\mathrm{A}| \mathrm{A}$..., where $\mid$ represents a layer of intercalant, the first order diffraction signal is expected to increase by a factor of $\sim 4$ while the second order signal remains unchanged [2]. Dark field STEM imaging can locate areas experiencing this shift as it occurs. Figure 1A illustrates the ideal imaging conditions, where only the first order Bragg reflections hit the annular dark field (ADF) STEM detector. Imaging GICs in this regime highlights changes in the graphite stacking order.

We mechanically exfoliated graphite, transferred chosen flakes onto instrumented, electrontransparent membranes (Fig. 1B), added $\mathrm{H}_{2} \mathrm{SO}_{4}$, and assembled vacuum-tight fluid cells for in situ STEM [3]. Figure 2 shows three ADF images taken from a 25 minute video of a graphite flake undergoing electrochemical intercalation. The graphite was cycled from $-16.4 \mathrm{mV}$, the open circuit potential (OCP), to $0.9 \mathrm{~V}$ relative to a platinum pseudo-reference electrode using a Gamry 600 potentiostat. The platinum counter and pseudo-reference electrodes are outside the field of view in Fig. 2 but visible in Fig. 1B.

Figure 2A shows a STEM image of a graphite flake at the OCP. Electrochemical intercalation of the graphite caused the contrast variations seen in Fig. 2B. Because the pixels in STEM images are acquired serially (here with a pixel dwell time of $65 \mu \mathrm{s}$ ), fast intercalation events give spatial discontinuities in the observed contrast. Figures $2 \mathrm{~B}-\mathrm{C}$ were acquired sequentially and provide an example of such an event. The STEM raster scanned left to right and then top to bottom. The top $\sim 25 \%$ of Figs. $2 \mathrm{~B}-\mathrm{C}$ are nearly identical, but at the time indicated by the red arrow in Fig. $2 \mathrm{C}$ the graphite abruptly shows contrast that is more uniform than in Fig. 2B, but much like that of Fig. 2A. The graphite returned to an approximation of its initial state almost instantaneously. 
The Fig. $2 \mathrm{C}$ inset shows the cyclic voltammogram section obtained simultaneously with the frames $2 \mathrm{~B}-\mathrm{C}$. Time advances to the left, and black dotted lines indicate frame boundaries. A distinct deintercalation peak is coincident with the contrast change in Fig. $2 \mathrm{C}$, showing a clear correlation between an electrical transport event and the structural changes in the graphite.

\section{References:}

[1] J. -M. Tarascon and M. Armand, Nature, 414 (2001) p. 359.

[2] B. Shevitski et al., Physical Review B 87 (2013) 045417.

[3] E. R. White et al., Appl. Phys. Express 4 (2011) 055201.

[4] This work was supported by NSF DMR-1206849, and in part by FAME, one of six centers of STARnet, a Semiconductor Research Corporation program sponsored by MARCO and DARPA. The authors acknowledge the use of instruments at the Electron Imaging Center for NanoMachines supported by NIH 1S10RR23057 and the CNSI at UCLA.
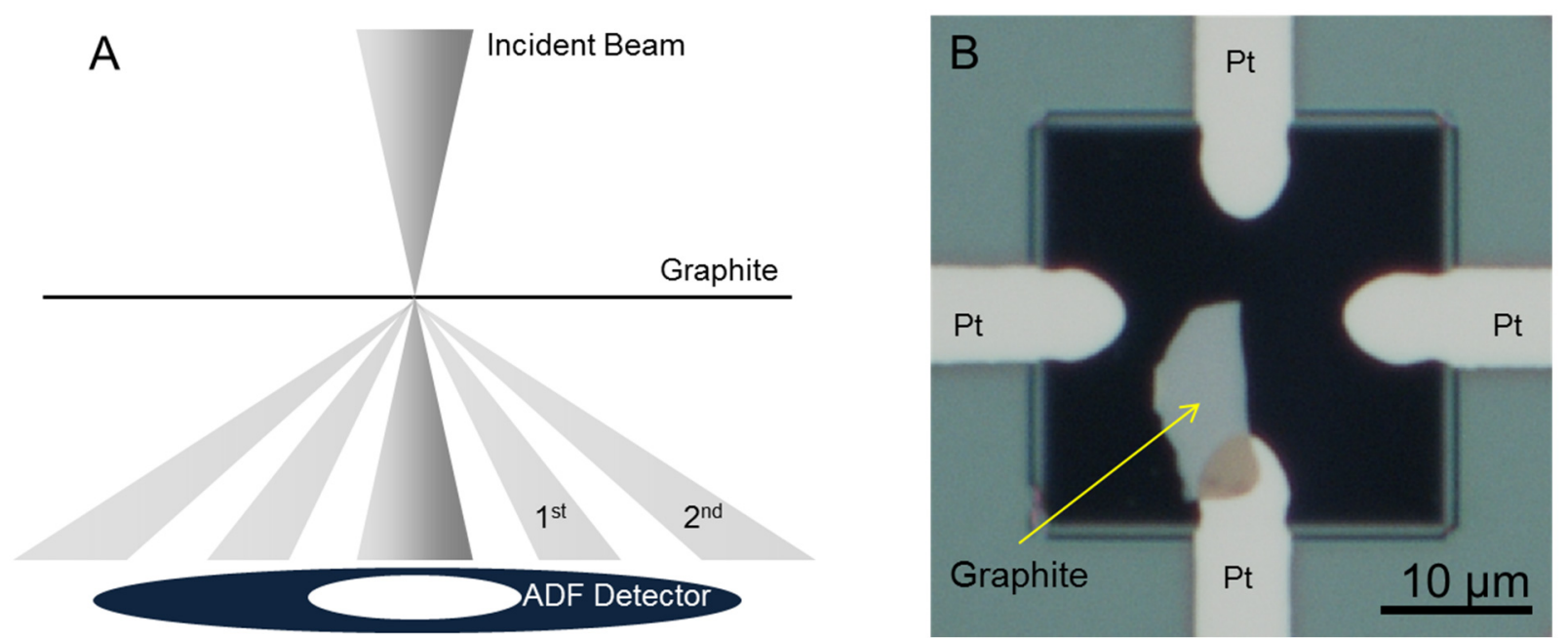

Figure 1. (A) ADF STEM conditions for capturing only the $1^{\text {st }}$ order Bragg reflections. The camera length is adjusted so that the central beam and the $2^{\text {nd }}$ order Bragg reflections miss the ADF detector. (B) A graphite flake is connected to the bottom platinum electrode on an electron transparent window. The other electrodes are used as counter and pseudo-reference electrodes.
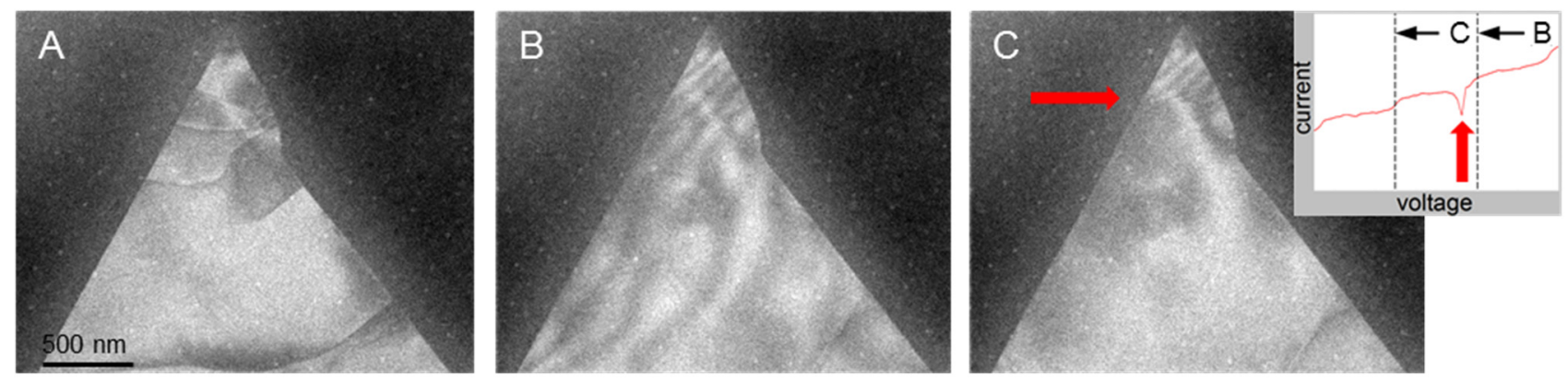

Figure 2. (A-C) ADF images of a single-crystal graphite flake intercalating and deintercalating for the second time. (A) Before intercalation. (B) Intercalating the graphite caused new contrast variations to appear. (C) The graphite sheet deintercalated at the point indicated by the red arrow. The inset shows the simultaneously-acquired electrical transport data. A (downward) current peak coincided with the contrast discontinuity in $(\mathbf{C})$. The scan rate was $2 \mathrm{mV} / \mathrm{s}$. 